\title{
Modulated Low Fault-Energy Protection Scheme for DC Smart Grids
}

\author{
Chunpeng Li, Puran Rakhra, Patrick Norman, Pawel Niewczas, Graeme Burt, Paul Clarkson
}

\begin{abstract}
DC smart grids enabled by the integration of advanced power electronic converters (PEC) can ease the integration and control of distributed renewable energy resources, electric vehicles and energy storage systems. However, these highly flexible power systems introduce many challenges when considering the design of reliable, plug-and-play protection that does not rely on dedicated communications infrastructure for device coordination. One particularly difficult challenge is the management of DC-side filter capacitor discharge during shortcircuit faults where the large peak fault-current produced can permanently damage exposed semiconductor components within the converter. One solution is to ensure that the trip-time of DC protection devices is sufficiently rapid (sub-millisecond) to guarantee that fault-current is blocked prior to reaching destructive magnitudes. However, such high-speed protection devices do not offer much margin for effective selectivity with downstream devices due to the narrow time window of operation. Accordingly, this paper proposes a non-unit protection scheme for future large-scale DC smart grid applications that increases this time-window of operation to enable improved selectivity whilst retaining a lower level of energy dissipated in the fault. Reliable protection coordination is demonstrated on a DC radial network and is realized using conventional millisecond trip-time devices, and a single solid-state microsecond trip-time device.

Index Terms-AC-DC power conversion, DC power systems, Power distribution faults, Power system protection, Power system simulation.
\end{abstract}

\section{INTRODUCTION}

$\mathrm{I}^{\mathrm{n}}$ creasing integration of renewable energy sources, electronic loads, energy storage systems (ESS), and electric vehicles in modern power networks is accelerating the deployment of lowvoltage DC distribution networks and microgrids [1]. DC power distribution provides a more efficient interface between supply and demand of energy in comparison to $\mathrm{AC}$ [1]. This is due to the reduction in end-to-end losses achieved by requiring fewer power conversion stages from the point of generation to the increasing quantity of highly efficient, electronically supplied DC loads and ESSs. The DC supply voltage can be tightly regulated via state-of-the-art PEC technology enabling highly stable DC systems that meet the strict power quality requirements of modern electronic appliances [2]. Accordingly, the PEC is the key enabler for the successful deployment of smart DC networks. In recent years, PEC efficiency has increased to a comparative level with similarly rated $\mathrm{AC}$ transformers, making modern DC networks a viable means of large-scale power distribution [3].

However, significant advancements in the development of standardized protection technologies for DC distribution systems are still required to ensure the safe and reliable deployment of DC networks. The integration of PECs with capacitive input and output filters can result in extremely highmagnitude transient currents in the event of DC network faults [4]. As significantly large fault current transients increase the risk of damaging vulnerable elements of PECs, it is commonly proposed that DC faults must be isolated prior to the transient peak using ultra-fast protection hardware [5].

Highly selective and high-speed differential [6] protection schemes have been demonstrated in advanced industrial applications such as aircraft [7], marine [8] and HVDC transmission [9] networks. However, these protection systems are not readily applicable to low-voltage large-scale DC distribution systems. To establish high-speed protection equipped with sufficient discrimination for such complex DC networks, a large quantity of high-speed protection devices are required. This includes advanced transducers, processors, solidstate circuit breakers, and precisely synchronized communication links. Additionally, if protection actions are executed only within a few samples, selectivity and stability may also be impaired.

The objective of these ultra-fast protection schemes is to prevent the converter diodes from being exposed to destructive fault-current levels. However, it is not necessary for the protection system to complete the fault isolation within this timeframe. In fact, it only requires a fast-acting current-limiter to retain low-energy current let-through in the event of a fault. Implementing a protection scheme that facilitates currentlimiting will allow the speed requirements for downstream discrimination to be relaxed. This strategy may be significantly advantageous for practical applications as it enables the use of conventional, plug-and-play overcurrent breakers for downstream protection. It also reduces hardware costs and improves protection sensitivity and stability compared to highspeed protection solutions.

This paper will propose a modulated low fault-energy (MLE) protection scheme which realizes graded overcurrent protection for DC distribution networks, utilizing a modulated solid-state fault current controller and conventional mechanical circuit breakers. Section II will review state-of-the-art LVDC protection schemes and present the challenges associated with practical implementation. Section III will present the algorithms of key components of the proposed MLE protection scheme and analyze power dissipation at the fault. Simulation results are presented in Section IV and are validated experimentally in Section V to show the effectiveness of MLE protection.

\section{LITERATURE REVIEW}

The dynamics of a DC power system under fault conditions define the requirements of its instrumentation and protection systems. Typical DC systems are commonly fed by conventional two-level voltage source converters (VSCs) that utilize relatively large DC-side filter capacitors. The rapid dissipation of the stored energy within these capacitors under short-circuit fault conditions is particularly challenging to protect against. Fig. 1 presents an equivalent model of a pole- 


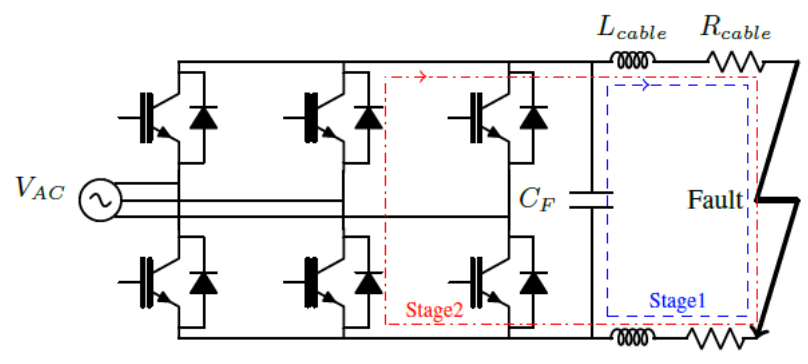

Fig. 1. Circulation stages of a VSC pole-to-pole fault.

to-pole low-impedance short-circuit fault in which the fault response can be divided into two stages [4]. During Stage 1, a high-magnitude current transient caused by the discharge of the capacitor occurs. After the capacitor voltage decreases to zero, the fault condition enters Stage 2 in which the high-magnitude fault current circulates through the antiparallel diodes within the converter. This behavior presents a significant risk of damage to VSCs if the fault is not removed from the circuit by the protection device prior to this stage. Accordingly, VSCbased LVDC networks require ultrafast protection to prevent permanent damage to power-electronic devices [4].

The majority of recently proposed protection schemes employ solid-state circuit breakers (SSCB) to realize highspeed protection in LVDC networks. The key principle is to minimize the fault detection and interruption time to prevent the converter diodes from being damaged by reversed fault current flow. These schemes can be categorized into overcurrent, differential and distance protection schemes.

High-speed overcurrent protection is achieved using a solidstate device in series with the VSC link capacitor. The SSCB will block the capacitor discharge in approximately $3-7 \mu$ s as the measured current exceeds a pre-set overcurrent threshold [10]. However, such rapid upstream protection may hinder protection coordination of downstream networks [7].

High-speed current differential protection provides effective selectivity by summing the boundary currents flowing into a protected area. The differential relay will operate immediately once the net-current exceeds a pre-set threshold. This method has been validated experimentally and can effectively discriminate and isolate faulted network sections within $<10 \mu \mathrm{s}$ [6]. Directional and regional differential protection can realize protection selectivity in a similar manner but relies on lower data throughput over communication channels and less SSCB hardware respectively. Directional protection only compares the directions of boundary currents of a trip-zone. An internal fault is detected when boundary currents flow inward [11]. Regional differential protection divides an LVDC network into medium-sized regions with SSCBs, whilst mechanical circuit breakers (MCB) are installed to protect smaller zones within each region. The SSCB will temporarily block fault current infeed using overcurrent protection, meanwhile the $\mathrm{MCB}$ detects the specific fault zone using differential protection. The SSCB will reconnect the power supply after the moderate-speed fault isolation of MCBs [12]. These current comparison-based protection schemes offer effective selectivity and high-speed performance. However, differential schemes fundamentally require high-bandwidth, highly-synchronized and reliable communication links. Furthermore, false-trips during external zone faults may occur if current measurements are not precisely synchronized [6].
High-speed distance protection is realized by deriving rateof-change measurements, such as $d i / d t$ [13], $d v / d t$ [14], and $d^{2} i / d t^{2}[15]$. These schemes can provide protection selectivity without the assistance of communications. Protection schemes that use such measurements have been demonstrated experimentally with fault detection and discrimination achieved within a few microseconds. However, real-world application of such advanced protection schemes may not be reliable. This is because rate-of-change measurements are very sensitive to noise [16].

Though the high-speed protection schemes effectively ensure low-energy dissipation during faults, these methods may be difficult to implement in practice [17]. High-speed protection requires ultra-fast critical hardware, such as SSCBs, communication links, transducers and processors. Use of these may increase cost and system complexity. This may be acceptable for advanced LVDC applications, such as aircraft/marine networks but may impede the commercialization of civil LVDC networks. Furthermore, rapid fault-isolation speeds may cause protection stability issues. As the protection time-window is restrained to sub-milliseconds, protection relays may be affected by external disturbances. For example, lightning strikes and EMI may result in a maloperation. This protection stability issue may be problematic in future large-scale LVDC networks.

Alternatively, fault current limiters (FCL) may be employed to avoid the need for ultrafast protection schemes. FCLs may be implemented using a full-bridge converter [18], superconducting fault current limiter [19], or solid-state fault current limiter [20]. Using such devices, downstream relays will gain a longer time-window to realize protection coordination with conventional MCBs. A method proposed by Qi in ABB Inc. utilized an upstream inductive solid-state FCL to limit fault current and downstream MCBs to select the fault location using differential/directional protection schemes [20]. The number of SSCBs needed is dramatically reduced, but comparison-based protection schemes still rely on communication systems. The 'FCL+MCBs' structure enables moderate-speed protection strategies, but the design of downstream MCB-relay coordination has not been considered in the literature.

Inspired by inverse definite minimum time (IDMT) protection in conventional AC systems, MLE protection enables the effective design of graded overcurrent protection for current-limited DC systems. This scheme controls the fault current let-through by modulating a solid-state SSCB whilst addressing the MCB-relay coordination problem using a derived numerical methodology.

\section{MLE PROTECTION CONCEPT}

The MLE protection scheme is composed of a single upstream SSCB and multiple downstream slower-acting MCBs. The SSCB and MCBs consist of a solid-state fault current controller (SSFCC) and associated MCB-relays respectively. The concept of the MLE protection scheme can be demonstrated on the example network shown in Fig. 2, and is described further in the following sections. 


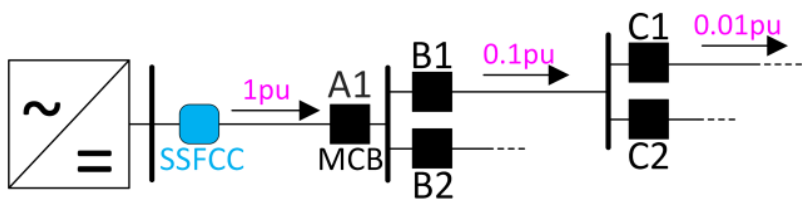

Fig. 2. Example network.

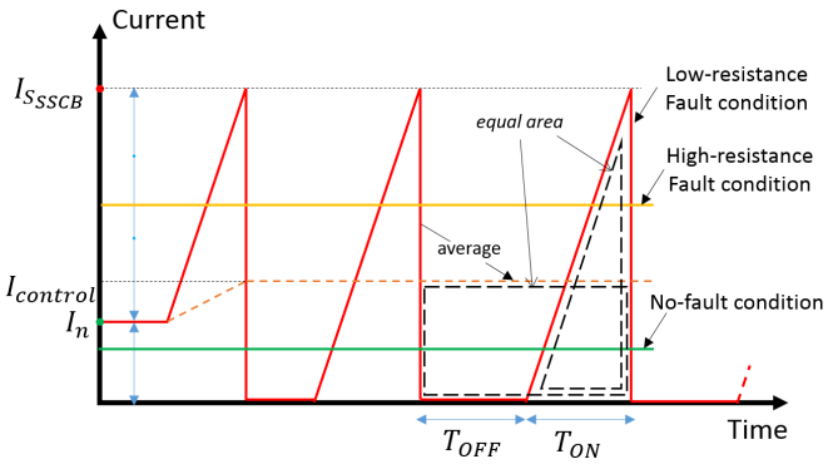

Fig. 3. Representation of the current profile associated with SSFCC control.

\section{A. Protection Algorithm of SSFCC}

The SSFCC is installed at the output terminal of the VSC capacitor. This device employs a high-frequency current transducer, a relay processor, and an SSCB. The SSFCC topology consists of two anti-series MOSFET or IGBT/diodepair devices enabling bidirectional current blocking. The operating principle of the SSFCC and expected current-limiting behavior is shown in Fig. 3. In the no-fault (NF) condition, where the current flow is no greater than the rated current of the network, the SSFCC will remain closed. If a high-resistance fault (HRF) occurs on the network, in which the resulting fault current moderately exceeds the nominal setting, the SSFCC will also remain closed and leave the downstream MCBs to trip on overcurrent. However, in the event of a low-resistance fault (LRF), where the current is detected to exceed a predefined 'high' threshold setting, the SSFCC will begin to control the fault current let-through by modulating. The duty-cycle of switching is actively controlled to constrain the average current to a predefined safe level that limits damage to components within the fault path. When the instantaneous current settles to non-fault levels, it indicates that one of the downstream MCBs has isolated the faulted section of the network. The SSFCC thereby remains closed to restore steady-state power to the system. However, if the SSFCC keeps modulating for an extended period, it indicates that all the downstream relays have failed to isolate the fault. Under these conditions, the SSFCC shall remain open to de-energize the network from this location as a failsafe coordinated backup. The response time of the SSCB is typically sub-microsecond and the conduction loss is normally negligible. Taking Semikron SKM 111AR MOSFET [23] as an example, the response time is as short as $270 \mathrm{~ns}$, and the typical drain-source resistance of two anti-series MOSFET/diode-pair is $14 \mathrm{~m} \Omega$. Accordingly, the short response time and low power loss will enable the use of the SSCB as a fault current limiter.
TABLE I. Example setting of SSFCC.

\begin{tabular}{|c|c|c|c|c|}
\hline$V_{n}$ & $I_{n}$ & $I_{S_{S S C B}}$ & $I_{\text {control }}$ & $T_{L}$ \\
\hline 1 & 1 & $4 I_{n}$ & $1.2 I_{n}$ & $1 \mathrm{sec}$ \\
\hline
\end{tabular}

Regarding the setting criteria, let $V_{n}$ and $I_{n}$ be the nominal voltage and current of the system; $I_{S_{S S C B}}$ is the modulating current threshold of the SSFCC; $I_{\text {control }}$ is the target average current let-through for LRF conditions; $T_{O N}$ and $T_{O F F}$ are the on and off times of the SSFCC during each duty-cycle; and $T_{L}$ is the failsafe modulating time before hard turn-off. Table I shows the relay settings of an example MLE configuration.

The nominal voltage $\left(V_{n}\right)$ and current $\left(I_{n}\right)$ are assumed to be unity. The modulating current threshold $\left(I_{S_{S S C B}}\right)$ must be set to ensure that transient fault currents do not damage downstream cables and components. The converter must therefore have sufficient overhead capacity to supply overloaded conditions. The target average current $\left(I_{\text {control }}\right)$ should be set to a safe level, but must be higher than the nominal current, to ensure that the downstream network voltage will recover after the fault is cleared (accounting also for the impact of constant power loads on network voltage restoration). $T_{L}$ must be set to ensure the downstream MCB-relays and backup devices have sufficient time to discriminate fault locations. Since $T_{O N}$ depends upon the fault-path circuit parameters, which are essentially uncontrollable, the moving average current is controlled by adjusting the off-time of the solid-state switch $\left(T_{O F F}\right)$.

The initial discharge current of every cycle can be approximated to a linear increment with a slope of $V_{n} / L_{f}$, where $L_{f}$ is the inductance of the fault path. Accordingly, the fault current during each on-period may be presented as

$$
i(t) \approx \frac{V_{n}}{L_{f}} t,
$$

where $t=0$ for each re-closing moment of the SSFCC. As the instantaneous current reaches the overcurrent threshold, the SSCB will open and block fault current. Substituting $i(t)=$ $I_{S_{S S C B}}$ in (1), the on-period becomes

$$
T_{O N} \approx \frac{I_{S S C B}}{V_{n} / L_{f}} .
$$

The off-period is controlled to limit the average fault current to $I_{\text {control }}$. Considering each modulation cycle in Fig. 3, equating the rectangular area by $I_{\text {control }}$ and the triangle area by fault current, such that

$$
\left(T_{O N}+T_{O F F}\right) \cdot I_{\text {control }} \approx \frac{1}{2} I_{S_{S S C B}} T_{O N},
$$

the off-period may be calculated as an equation, that is

$$
T_{O F F} \approx \frac{1}{2}\left(I_{S_{S S C B}} T_{O N} / I_{\text {control }}\right)-T_{O N} .
$$

\section{B. Protection Algorithm of MCB-Relays}

For conventional electromechanical and microprocessorbased AC relays, the IDMT characteristics are derived from a formula that complies with BS142 and IEC 60255 standards. It is generally defined as [21]

$$
t_{\text {trip }}=\frac{K}{\left(\frac{I_{f}}{P S \cdot I_{S}}\right)^{\alpha}-1} \times T M S,
$$

where $t_{\text {trip }}$ is the trip-time; TMS is the time multiplier setting; $I_{f}$ is the RMS-value of AC fault current; $I_{S}$ is the value of relay 
current setting; $P S$ is the relay plug setting; $\alpha$ and $K$ are constants.

A DC equivalent version of an IDMT protection scheme can be applied to the downstream MCB-relays within this system. As the SSFCC will limit fault current to a level lower than the trip threshold, $I_{S_{S S C B}}$, the very inverse characteristic curve [21] is selected for such systems, where $\alpha$ is unity. Hence, the MCB-relay trip characteristic becomes

$$
t_{\text {trip }}=\frac{K T M S}{\frac{\overline{I_{f}}}{P S \cdot I_{M C B}}-1},
$$

where $K$ and TMS are combined as one setting, KTMS; $\bar{I}_{f}$ is the moving average value of DC fault current; and $I_{S_{M C B}}$ is the overcurrent threshold setting of the MCB. For each MCB-relay, $I_{S_{M C B}}$ is normally set marginally higher than the nominal load current flowing through this branch, e.g. 1.2I $I_{n}$, and KTMS and $P S$ are adjustable to realize protection coordination.

Since the fault current will be modulated during an LRF condition, MCB-relays will require a means to compute the moving average current value and execute the protection algorithm. This may be implemented using DSP hardware. Equation (6) can be presented in a numerical form, such that

$$
\text { inc }=\left(\frac{\bar{I}_{f}}{P S \cdot I_{S_{M C B}}}-1\right) \cdot \frac{C_{T} \cdot T_{S_{M C B}}}{K T M S},
$$

and

$$
t_{\text {trip }}=\frac{C_{T}}{i n c} \cdot T_{S_{M C B^{\prime}}}
$$

where $T_{S_{M C B}}$ is the sampling time of the numerical relay; $C_{T}$ is the counting threshold; and inc is the incremental value at every sample. The trip-time is affected by the KTMS and $P S$ settings only, and is independent of $T_{S_{M C B}}$ and $C_{T}$ settings, as evident in equations (7) and (8).

The KTMS setting enables the grading of trip-times between MCB-relays at different downstream locations, whilst the $P S$ setting enables the coordination of trip-times between LRF and $\mathrm{HRF}$ conditions of an individual MCB-relay. Hence, $P S$ is a voltage-controlled setting which is unity in NF and HRF conditions, and equal to a voltage-dependent coefficient, $k_{V_{d e p}}$, in LRF conditions. Accordingly, $P S$ may be expressed as

$$
P S=\left\{\begin{array}{cc}
1, & V_{M C B}>0.8 V_{n} \\
k_{V_{\text {dep }}}, & V_{M C B}<0.8 V_{n}
\end{array}\right.
$$

where $V_{M C B}$ and $V_{n}$ are the measured local voltage and nominal local voltage respectively.

\section{1) Protection strategy for high-resistance or overloading} faults

Fig. 4 shows a schematic of a representative DC system to demonstrate the operation of the MLE protection strategy for high-resistance or overloading faults. At any time, the current flowing through any MCB-relay may be defined as I(local), whilst the sum of the nominal currents of all other branches may be defined as $I_{n}$ (other). During HRF conditions, such as that shown in Fig. 4, the current through all upstream MCB-relays will exceed their respective trip thresholds. However, the current through the SSFCC will be insufficient to trigger its modulation action. i.e. $I_{S_{M C B}}<I$ (local) $<I_{S_{S S C B}}-$ $I_{n}$ (other). Under such conditions, the network will remain at its nominal voltage, and so the voltage-controlled $P S$ setting will remain at unity according to equation (9).

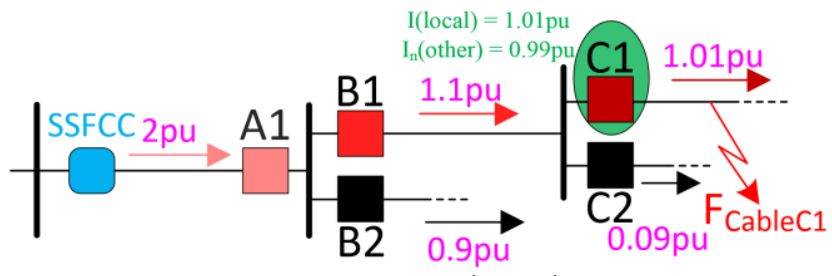

Fig. 4. Schematic of $I$ (local) and $I_{n}$ (other) of an MCB-relay.

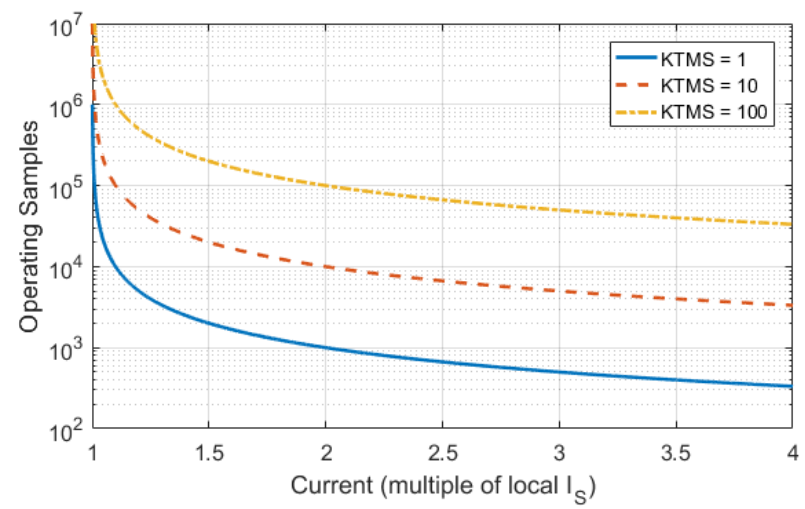

Fig. 5. Relationship between $M_{f}$ and $N_{\text {trip }}$.

Substituting $P S$ in equation (6) with this value and defining $I$ (local) $/ I_{S_{M C B}}$ as $M_{f}$ (which represents the multiple of the local MCB trip threshold), an overcurrent condition can be signified when $M_{f}>1$. Rearranging equation (6) and defining $t_{\text {trip }} / T_{S_{M C B}}$ as $N_{\text {trip }}$, the trip-time in terms of the number of samples becomes

$$
N_{\text {trip }}=\frac{K T M S}{T_{S_{M C B}\left(M_{f}-1\right)}} .
$$

The inversely-proportional relationship between $N_{\text {trip }}$ and $M_{f}$ is presented in Fig. 5, where $T_{S_{M C B}}$ is assumed to be $1 \mathrm{~ms}$. Therefore, in the HRF fault condition, trip-times can be adjusted by varying only the KTMS setting of each MCB-relay to realize protection coordination.

\section{2) Protection settings for short-circuit or low-resistance} faults

In the event of low-resistance or short-circuit faults (LRF condition), where the fault resistance is below a critical value $\left(R_{f_{C}}\right)$, the SSFCC will begin to modulate and regulate the moving average current to $I_{\text {control }}$. The critical fault resistance may be defined as

$$
R_{f_{C}}=\frac{V_{n}}{I_{S S S C B}-I_{n}} .
$$

Since the fault path is a low-resistance network with a low average fault-current, the voltage of the system will collapse. The collapsed local voltage is thereby a Boolean metric for an MCB-relay $P S$ setting to transition to the LRF state.

The voltage-dependent coefficient $\left(k_{V \text { dep }}\right)$ of a MCB-relay is a value from 0 to 1 , enabling a faster trip-speed in comparison to HRF condition trip-speeds. Under the LRF condition, the MCB-relay compares its local moving average current with a reduced current threshold, due to the use of $k_{V \text { dep }}$. Accordingly, the multiple of the local MCB trip threshold, $M_{f}$, becomes $I$ (local) $/\left(k_{V \text { dep }} \cdot I_{S_{M C B}}\right)$. Substituting this in equation (10) will enable protection coordination for low-resistance faults to be realized by adjusting KTMS. 
The coefficient $k_{V_{\text {dep }}}$ may be tuned to obtain the desired local trip-time for each MCB under LRF conditions. A lower value of $k_{V_{\text {dep }}}$ will enable a faster MCB trip response, however, a minimum time margin between coordinating devices must be kept to ensure effective selectivity. Too rapid a trip-time may reduce this time margin by causing upstream MCBs to be excessively sensitive to fault current. This may result in false-tripping, impacting the stability and security of the protection system.

Furthermore, considering that the network voltage is collapsed and that all loads are de-energized, the trip-time under low-resistance fault conditions should be selected such that it is no greater than the minimum trip-time under high-resistance fault conditions. This minimum trip-time occurs when the equivalent network-resistance, during a high-resistance fault, approaches the critical fault resistance $\left(R_{f_{c}}\right)$ [22] of the system.

Accordingly, $k_{V \text { dep }}$ may be optimally tuned such that the trip-time under critical high-resistance faults (nominal voltage sustained) and low-resistance faults (network voltage collapsed) is continuous. In the event of an HRF where voltage is sustained, the local MCB current will be in the range of

$$
I_{S_{M C B}}<I(\text { local })<I_{S_{S S C B}}-I_{n}(\text { other }),
$$

This range of local MCB current measurement can be rearranged to represent $I$ (local) in terms of a multiple of its trip threshold, $M_{f}$, such that

$$
1<M_{f}(H R F)<\frac{{ }^{I_{S S S C B}}-I_{n}(\text { other })}{I_{S_{M C B}}} .
$$

During LRF conditions where voltage is collapsed, I(local) becomes $I_{\text {control }}$. Accordingly, $M_{f}$, under these conditions can be derived as

$$
M_{f}(L R F)=\frac{I_{\text {control }}}{I_{S_{M C B} \cdot k_{V d e p}}} .
$$

Equating the maximum $M_{f}(H R F)$ and $M_{f}(L R F), k_{V d e p}$ can be derived as

$$
k_{V_{\text {dep }}}=\frac{I_{\text {control }}}{I_{S_{S S C B}-I_{n}(\text { other })}} .
$$

When configuring the MLE protection scheme for a DC distribution network, appropriate values of KTMS must be assigned to coordinate trip-times between each MCB-relay. The principle for assigning KTMS in terms of its target trip-time can be obtained by re-arranging equations (6), where

$$
K T M S=T_{\text {trip }}\left(\frac{I(\text { local })}{k_{V_{\text {dep }}{ }^{I} S_{M C B}}}-1\right) .
$$

\section{Protection System States}

The behavior of the protection system during NF, LRF and HRF states is summarized in Table II. To configure the MLE protection system for the DC network in Fig. 2, Fig. 6 illustrates the characteristic between the trip-times in terms of samples and the local average current measurement of different $\mathrm{MCB}$ current ratings. In this example, all KTMS are set to unity; $I_{S_{M C B}}$ of each relay are set 1.2 times its local nominal current; $T_{S_{M C B}}$ is set to $1 \mathrm{~ms}$; and $k_{V_{d e p}}$ is set by equation (15).

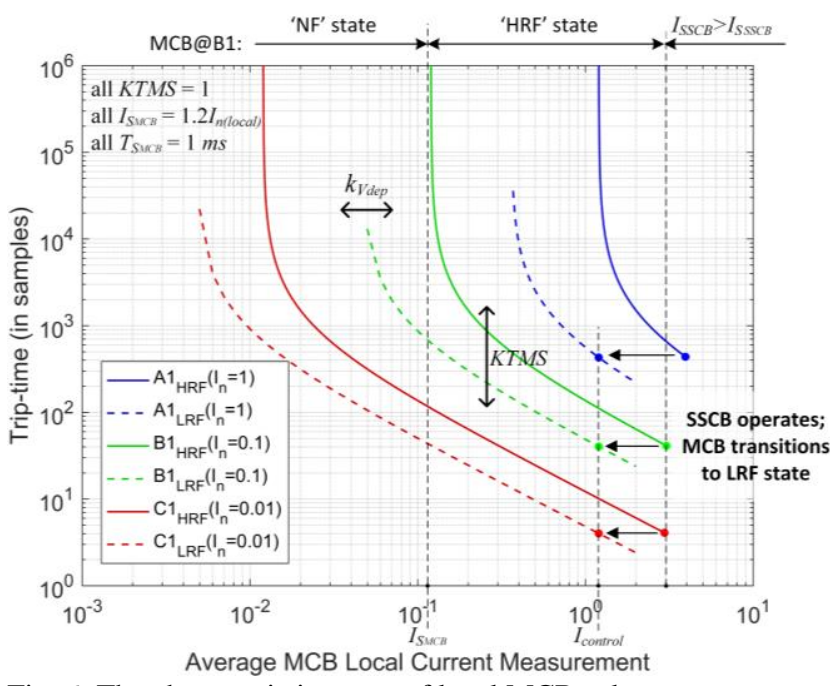

Fig. 6. The characteristic curve of local MCB-relays.

TABLE II. Protection System States

\begin{tabular}{|c|c|c|c|c|}
\hline $\begin{array}{c}\text { Protection } \\
\text { System } \\
\text { States }\end{array}$ & \multicolumn{2}{|c|}{ SSFCC } & \multicolumn{2}{c|}{ MCB-Relay } \\
\cline { 2 - 5 } & Behaviour & Current Range & $\begin{array}{c}\text { Average } \\
\text { Voltage }\end{array}$ & Behaviour \\
\hline $\mathrm{NF}$ & Stable & $I \leq I_{n}$ & Normal & Reset counter \\
\hline $\mathrm{HRF}$ & Stable & $I_{n}<I<I_{S_{S S C B}}$ & Normal & $\begin{array}{c}\text { Trip until } \\
\text { counter full }\end{array}$ \\
\hline LFR & Modulating & $\bar{I} \cong I_{\text {control }}$ & Collapsed & $\begin{array}{c}\text { Decrease } I_{S}, \\
\text { trip until } \\
\text { counter full }\end{array}$ \\
\hline
\end{tabular}

Taking Relay B1 as an example, the characteristic curve in Fig. 5 indicates actions of different MCB-relays in the three protection states. When the local current through an MCB is measured to be under its overcurrent setting, the MCB will remain stable. When the local current exceeds the MCB overcurrent setting, and the total current through the SSFCC is less than the modulation threshold that triggers current limiting, the MCB-relay will operate in HRF state. As shown in Fig. 6, MCBs with low nominal current ratings are more sensitive to overloading. Accordingly, selective protection coordination may be deployed that includes effective failsafe backup protection that is graded in a deterministic manner. When the total current reaches the SSFCC current threshold, the modulation action will be triggered and average current will be regulated to a reduced level. This will cause the network voltage to collapse, triggering all MCBs to transition into the LRF state. The MCB-relay will then operate on the LRF curve and operate at the point of $I_{\text {control }}$.

Due to the optimally tuned $k_{V d e p}$ coefficient, the MCB triptime for a low-resistance fault is designed to be equal to its local minimum trip-time under the HRF state. This will maintain the effective coordination and backup functionality of the protection system when operating in the LRF state.

\section{Analysis of Power Supplied to the Fault}

In the HRF state, the SSFCC will remain on and the network voltage will remain at nominal levels. Accordingly, power dissipated in the fault can be derived, where

$$
P_{f}=V_{n}^{2} / R_{f} \text {. }
$$


In the LRF state, with the SSFCC modulating, the power dissipated in the fault may be determined by analyzing the energy dissipated in each-cycle divided by the period of each cycle, where

$$
P_{f}=\frac{E_{c y c l e}}{T_{c y c l e}}=\frac{\int_{0}^{T} O N_{i^{2}}(t) R_{f} d t}{T_{O N}+T_{O F F}} .
$$

Substituting (1), (2) and (4) into (18), the fault power can be derived, where

$$
\begin{aligned}
P_{f} \approx & \frac{\int_{0}^{\frac{I_{S S S C B}}{V_{n} / L_{f}}}\left(V_{n} / L_{f}\right)^{2} t^{2} R_{f} d t}{\frac{1}{2} I_{S_{S S C B}} \cdot \frac{I_{S S C B}}{V_{n} / L_{f}} / I_{\text {control }}} \\
= & \frac{2}{3} R_{f} I_{S_{S S C B}} I_{\text {control }} .
\end{aligned}
$$

Combining (17) and (19),

$$
P_{f}=\left\{\begin{array}{c}
V_{n}^{2} / R_{f}, R_{f_{c}}<R_{f}<+\infty \\
\frac{2}{3} R_{f} I_{S_{S S C B}} I_{\text {control }}, 0<R_{f} \leq R_{f_{c}}
\end{array} .\right.
$$

In equation (20), it can be observed that $P_{f}$ is inverselyproportional to $R_{f}$ in the HRF state, and proportional to $R_{f}$ in the LRF state. The maximum fault power occurs when $R_{f}$ is approximately equal to $R_{f_{c}}$, and is different for both cases due to the SSCB current limiting operation.

Accordingly, for the case study presented in this paper, where $I_{S_{S S C B}}$ is set to 4 p.u., and $I_{\text {control }}$ is set to $1.2 p . u$., the highest fault power in the HRF state is $3 p . u$., and 1.07 p.u. in the LRF state. This is potentially an order of magnitude lower than if a non-MLE protection method is used.

\section{VERIFICATION OF MLE OPERATION WITH SIMULATION}

The effectiveness of the MLE protection scheme is verified through simulation using a model of an example DC network represented in Fig. 7. This model consists of one SSFCC at position $\mathrm{A} 1$ and $5 \mathrm{MCBs}$ at downstream feeder locations that operate in coordination to provide backup protection in both HRF and LRF states. The SSFCC settings are defined in TABLE I on page 3, whilst the MCB-relay settings are described in TABLE III.

In TABLE III, $T_{\text {trip }}$ (target) is the target trip time of each relay in the LRF state. $k_{V d e p}$ is set by equation (15), and KTMS is set by equation (16). The time margin of each target trip time is reserved to $20 \mathrm{~ms}$, which must be set higher than the MCB interruption time to avoid mis-coordination.

Taking an LRF case as an example, Fig. 8 presents the instantaneous current responses measured at Relay A2, during the initiation of the fault. The current is modulated, and the length of off-time in each cycle is adjusted by the SSFCC to achieve the steady-state moving average current, $I_{\text {control }}$. Fig. 9 presents the actions of Relay A2. As shown in Fig. 9 (a), the moving average current is controlled to approximately $1.2 I_{n}$, whilst the average voltage is almost zero. As shown in Fig. 9 (b), the relay will calculate the increment, inc, every $1 \mathrm{~ms}$ according to equation (7) and (9). The number in the counter will accumulate each increment value, as shown in Fig. 9 (c), until it exceeds the counter threshold setting, $C_{T}$ triggering the trip signal as shown in Fig. 9 (d). The results indicate that the actual trip time is the same as the target trip time shown in TABLE III.

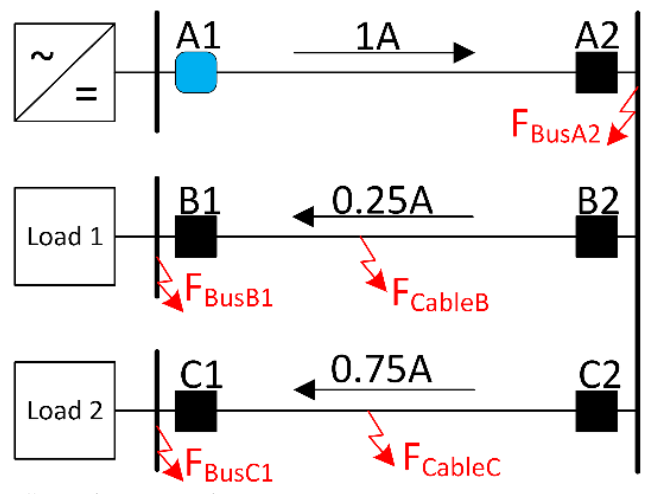

Fig. 7. Sample network.

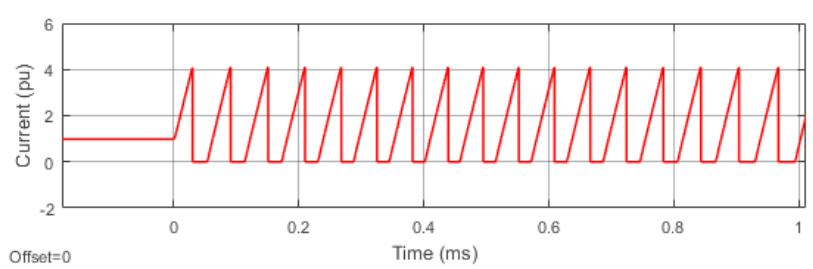

Fig. 8. Instantaneous responses of Relay A2 under LRF condition
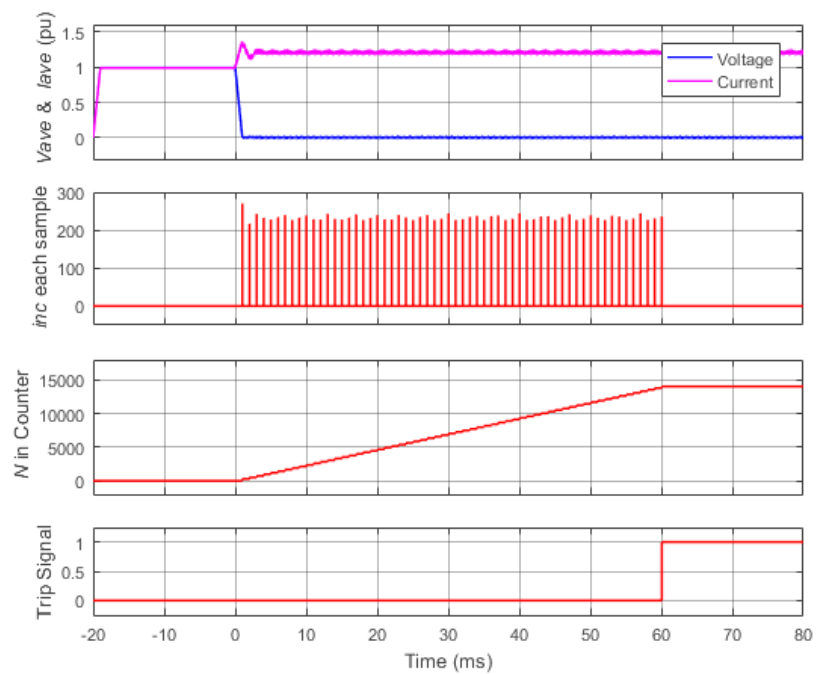

Fig. 9. Actions of Relay A2, a) moving average voltage and current, b) the increment of each sample, c) the accumulated number in the counter, d) trip signal.

TABLE III. Example setting of MCB-relays.

\begin{tabular}{|c|c|c|c|c|c|}
\hline & B1 & B2 & C1 & C2 & A2 \\
\hline$T_{S_{M C B}}$ & \multicolumn{5}{|c|}{$1 \mathrm{~ms}$} \\
\hline$I_{n}$ & 0.25 & 0.25 & 0.75 & 0.75 & 1 \\
\hline$I_{S_{M C B}}$ & 0.3 & 0.3 & 0.9 & 0.9 & 1.2 \\
\hline$k_{\text {Vdep }}$ & 0.37 & 0.37 & 0.32 & 0.32 & 0.3 \\
\hline$T_{\text {trip }}($ target $)$ & $20 \mathrm{~ms}$ & $40 \mathrm{~ms}$ & $20 \mathrm{~ms}$ & $40 \mathrm{~ms}$ & $60 \mathrm{~ms}$ \\
\hline KTMS & 0.196 & 0.393 & 0.063 & 0.126 & 0.140 \\
\hline
\end{tabular}

As shown in Fig. 7, multiple fault scenarios are evaluated with variable fault resistances from 1 to $1000 \mathrm{~m} \Omega$ at different locations. Breaker operations are disabled so that the trip-time of backup MCB-relays can be observed. The trip-time of the main relays in each fault scenario is recorded in TABLE IV. Additionally, in order to demonstrate protection coordination, the trip-time of the main relay and backup relays for each fault scenario is plotted in Fig. 10. The power dissipated in each fault scenario is shown in Fig. 11. 
TABLE IV. Trip-time of the main protection MCB-relay for variable resistance faults at each position.

\begin{tabular}{|c|c|c|c|c|c|}
\hline Fault Pos. & BusB1 & CableB & BusC1 & CableC & BusA2 \\
\hline Main Relay & B1 & B2 & C1 & C2 & A2 \\
\hline$R_{f}(\mathrm{~m} \Omega)$ & \multicolumn{5}{|c|}{ Trip-time (ms) } \\
\hline 1 & 22 & 42 & 22 & 42 & 62 \\
\hline 2 & 22 & 42 & 22 & 42 & 62 \\
\hline 10 & 22 & 42 & 22 & 42 & 62 \\
\hline 30 & 22 & 43 & 22 & 42 & 62 \\
\hline 100 & 23 & 45 & 22 & 43 & 62 \\
\hline 200 & 25 & 48 & 23 & 44 & 62 \\
\hline 300 & 25 & 50 & 22 & 44 & 61 \\
\hline 330 & 22 & 42 & 22 & 43 & 64 \\
\hline 340 & 22 & 44 & 22 & 44 & 66 \\
\hline 400 & 26 & 51 & 26 & 52 & 78 \\
\hline 600 & 39 & 76 & 40 & 80 & 122 \\
\hline 800 & 52 & 102 & 55 & 110 & 171 \\
\hline 1000 & 65 & 129 & 72 & 143 & 223 \\
\hline
\end{tabular}

Fig. 10 shows that when the fault resistance is lower than the critical resistance (which in this case is $333.3 \mathrm{~m} \Omega$ ), the protection system operates in the LRF state where the trip-time remains nearly constant. When the fault resistance is greater than the critical resistance, the protection system operates in the HRF state where the trip-times of all the MCBs gradually increase as a function of higher resistance. Fig. 11 illustrates the fault power in terms of fault resistance at different locations. It indicates that the peak fault power is limited to $3 p . u$., and occurs at the critical resistance point.

\section{HARDWARE IMPLEMENTATION OF MLE PROTECTION}

This section presents the experimental validation of the MLE protection scheme on a low-voltage DC distribution system within a laboratory environment, as shown in Fig. 12. Details of the experimental hardware are provided in TABLE V. The DC distribution system has been configured to form the same architecture as illustrated in Fig. 7. The system is equipped with distributed voltage and current transducers, and solid-state switches to perform protection actions. A VSC is connected at

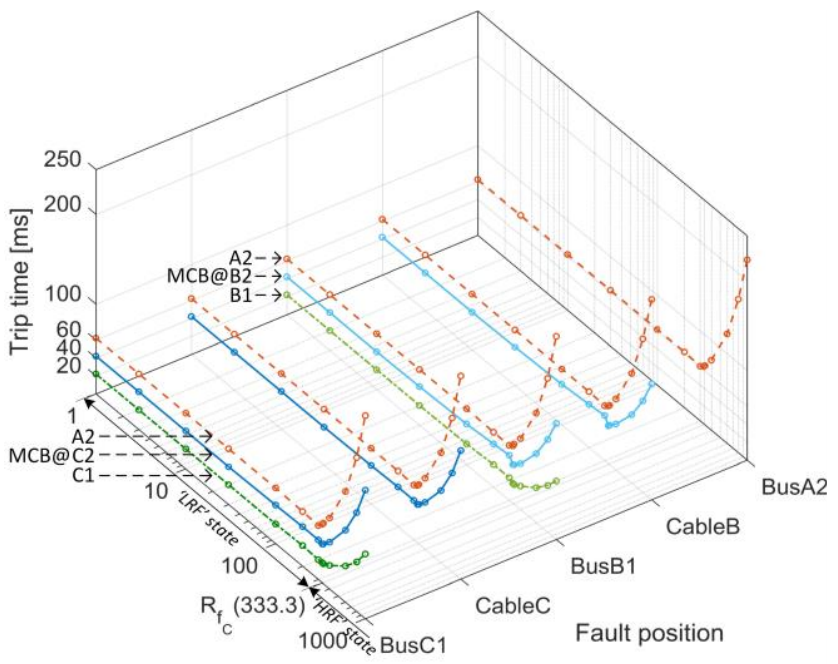

Fault resistance $[\mathrm{m} \Omega]$

Fig. 10. Result of protection trip-time and backup trip-time.

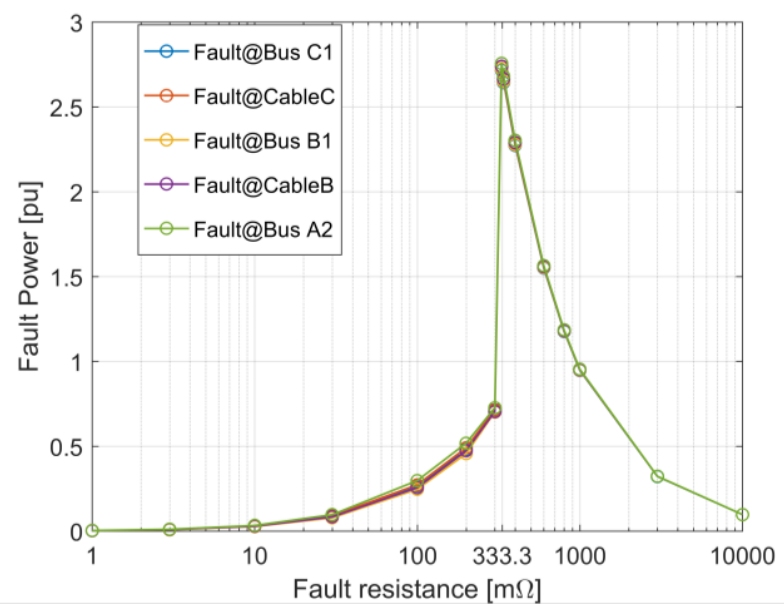

Fig. 11. Fault power dissipation.

Bus A1 supplying power to a $6.6 \Omega$ load located at Bus $\mathrm{C} 1$. The switch by Bus A1 employs the SSFCC algorithm whilst all other downstream switches apply the MCB-relay algorithm, emulating electromechanical protection devices. Data from the transducers is acquired centrally as shown in Fig. 13.

The central processor employs an FPGA-based controller which is used to capture measurement data from transducers and to control the power switches. Each switch is controlled independently based on its local voltage and current measurements. The SSFCC at A1 executes its protection function with a $1 \mathrm{MHz}$ high-speed control loop, while the MCB-relays conduct the protection algorithm with a $1 \mathrm{kHz}$ lowspeed loop using a moving average of the current profile.

The virtual relays employed on the FPGA controller are programmed according to the proposed setting strategy, however, due to hardware limitations, the SSFCC employs a fixed open-time in each duty-cycle rather than controlling the output current with a dynamic open-time. Accordingly, this is a preliminary qualitative experiment verifying the effectiveness of protection coordination using the MLE approach.

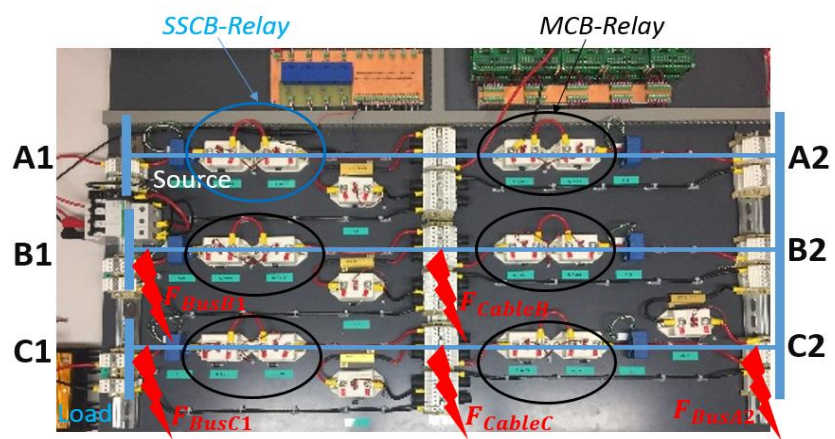

Fig. 12. DC rig setup.

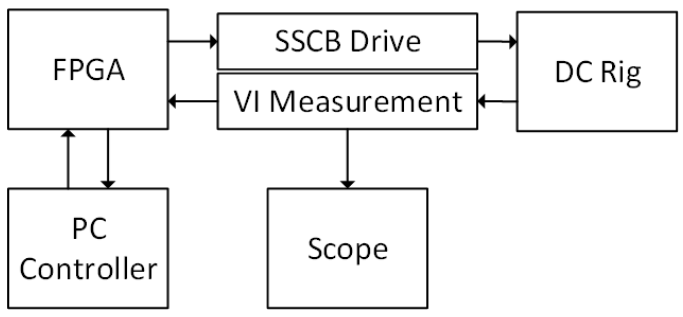

Fig. 13. Experiment layout. 
TABLE V. Details of Experimental Hardware

\begin{tabular}{|c|c|c|c|}
\hline & Function & Hardware & $\begin{array}{l}\text { Experiment test } \\
\text { settings }\end{array}$ \\
\hline 1 & Power supply & $\begin{array}{c}\text { MDL TOE-7621 320W } \\
100 \mathrm{kHz} \text { DC power } \\
\text { supply }\end{array}$ & $\begin{array}{c}\text { Set to } 24 \mathrm{~V} \text { constant } \\
\text { voltage }\end{array}$ \\
\hline 2 & $\begin{array}{l}\text { Disconnect supply } \\
\text { prior to fault }\end{array}$ & $\begin{array}{l}\text { Semikron SKM 111AR } \\
\text { MOSFET [23] }\end{array}$ & $\begin{array}{l}100 \mathrm{~V} \text { nominal, } 200 \mathrm{~A} \\
\text { nominal (600A max) }\end{array}$ \\
\hline 3 & Current measurement & LEM HAS 200-S [24] & $\begin{array}{l}50 \mathrm{~A} / \mathrm{V} \text { measurement } \\
\text { ratio }\end{array}$ \\
\hline 4 & Voltage measurement & LEM LV 25-P [24] & $\begin{array}{c}5.7 \mathrm{~V} / \mathrm{V} \text { measurement } \\
\text { ratio }\end{array}$ \\
\hline 5 & $\begin{array}{l}\text { Representative cable } \\
\text { inductor }\end{array}$ & Murata $15222 \mathrm{c}$ & $\begin{array}{l}2.2 \mathrm{uH}+/-20 \%, \\
4.2 \mathrm{mOhm}\end{array}$ \\
\hline 6 & Representative load & $\begin{array}{l}\text { Panel Mount Fixed } \\
\text { Resistor } \\
\end{array}$ & $6.6 \mathrm{Ohm}$ \\
\hline 7 & FPGA Processor & NI cRio-9024 [25] & \multirow{4}{*}{$\begin{array}{l}\text { Control loop time is } \\
1 \mu \mathrm{s}\end{array}$} \\
\hline 8 & Analogue input & Ni 9223 [25] & \\
\hline 9 & Analogue output & NI 9269 [25] & \\
\hline 10 & Digital I/O & NI 9401 [25] & \\
\hline 11 & Signal capture & $\begin{array}{c}\text { Tektronix OSC MSO } \\
\text { 2004B } \\
\end{array}$ & 1GS/s/channel \\
\hline
\end{tabular}

As shown in Fig. 7, rail-to-rail short-circuit faults are sequentially injected at Bus C1, Cable C, Bus B1, Cable B, and Bus A2, respectively to verify the performance of protection discrimination. Additionally, voltage at Bus A1 is continually monitored to ensure the link voltage of the DC source does not decrease to zero.

TABLE VI shows the trip-position and trip-time results for the short-circuit faults at different positions. The results indicate that only the nearest upstream MCB-relay will act to isolate the fault, as desired, and that the trip-time is graded to provide effective backup protection.

Fig. 14 presents voltage and current waveforms measured at Bus A1 when the fault is applied. As a fault occurs on the network, the SSFCC at Bus A1 starts to modulate the current causing the voltage of the VSC to be maintained above zero, intrinsically protecting the anti-parallel diodes. The average current is limited to non-detrimental levels, enabling the slower downstream MCBs to operate in coordination. Additionally, the modulated fault current provides zero-current periods which improves the reliability of MCBs when interrupting fault current.

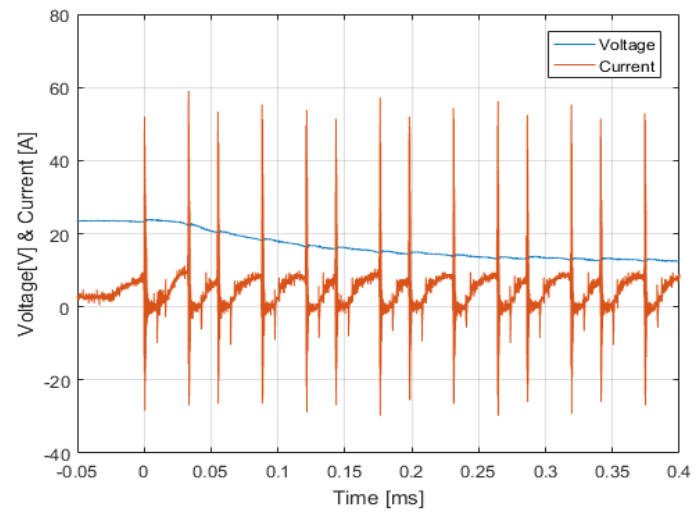

Fig. 14. Voltage and current waveform under a short-circuit fault.

TABLE VI. Protection Operation Results

\begin{tabular}{|c|c|c|c|c|c|}
\hline Fault position & Bus B1 & Cable B & Bus C1 & Cable C & Bus A2 \\
\hline Trip-position & B1 & B2 & C1 & C2 & A2 \\
\hline Trip-time & $1 \mathrm{~ms}$ & $5 \mathrm{~ms}$ & $1 \mathrm{~ms}$ & $5 \mathrm{~ms}$ & $8 \mathrm{~ms}$ \\
\hline
\end{tabular}

As observed in Fig. 14, large current transients are produced when the SSFCC at Bus A1 interrupts the current. They can be restrained by connecting a snubber in parallel with the SSCB. Nevertheless, these transients will not impact the coordinated operation of MCBs because the moving average measurement is not dramatically affected by the transient current.

\section{MLE APPLICATIONS IN OTHER GRID CONFIGURATIONS}

The MLE protection scheme has been demonstrated on a passive radial DC grid in the previous sections, however, renewable energy generation is widely used in LVDC distribution networks and some applications may adopt looptype DC grids. These applications may cause the fault current to be supplied from several sources, or in the case of a looptype network, from two fault paths. For LRF conditions, it is expected that directional MCB-relays can be utilized to achieve fast and coordinated fault isolation. However, for some HRF conditions, the measured fault current through the MCB-relays may actually be lower than the nominal rated current. To ensure the MCB-relay can operate with the designed speed, the following two solutions are proposed.

\section{A. Application in Loop-Type DC Grids}

\section{1) Equivalent model method}

The MLE protection scheme is feasible for protecting looptype DC grids using an equivalent model method. As shown in Fig. 15 (a), the current fed into Node $\mathrm{A}$ is equivalent to the summation of the clockwise and anticlockwise currents, $I_{c w}$ and $I_{a-c w}$. Using Kirchhoff's current law, if $I_{c w}$ is measured and then added to the measured current at each relay using a dedicated communication system, the loop-type DC grid can be equivalent to a radial grid as shown in Fig. 15 (b). Accordingly, the settings of each relay can be determined using the MLE method to realize coordinated protection in the anticlockwise direction. For example, if a fault occurs at Bus D in Fig. 15 (b), Relay D1 will provide primary protection, whilst Relay C2, C1, B2, B1 and A will provide backup protection.

The protection coordination in the clockwise direction can be realized in the same manner. $I_{a-c w}$ may be added to each relay to realize an equivalent clockwise radial network. Accordingly, each relay will have two settings and will operate based on the equivalent current direction.

\section{2) Alternative to Communication}

As communications may increase the cost and complexity of LVDC protection for the loop-type topology, two mechanical reclosers may be employed to temporarily interrupt the fault current on each path and realize coordinated protection in clockwise and anticlockwise direction successively.

As shown in Fig. 16, the two reclosers are installed at Bus A and controlled in terms of the current measurement at Relay A. If an overcurrent is detected at Relay A, one of the reclosers will be opened immediately so that the loop-type grid is reconfigured to form a radial network. The MCB-relays will operate in coordination in terms of the MLE protection scheme.

After the overcurrent through Relay A is cleared, the first recloser is closed again and the other recloser is opened to enable the coordinated protection in the other direction in the same manner. When the fault is isolated through both fault paths, both reclosers are returned to the on position to reinstate the power supply. 


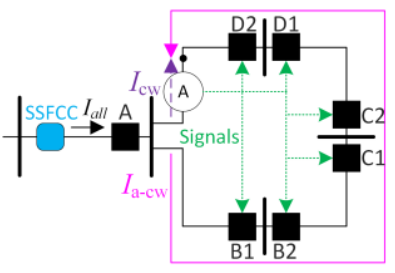

a) loop-type DC grid

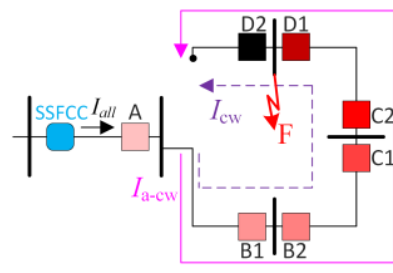

b) equivalent radial $D C$ grid
Fig. 15. Equivalent model method in loop-type DC grids.

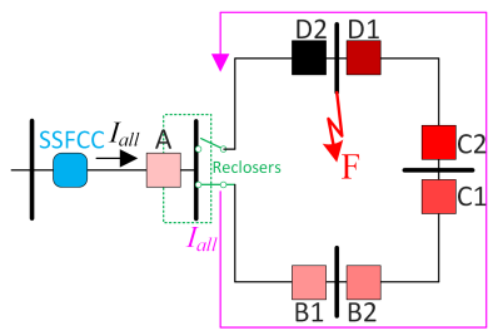

Fig. 16. Equivalent model method with reclosers.
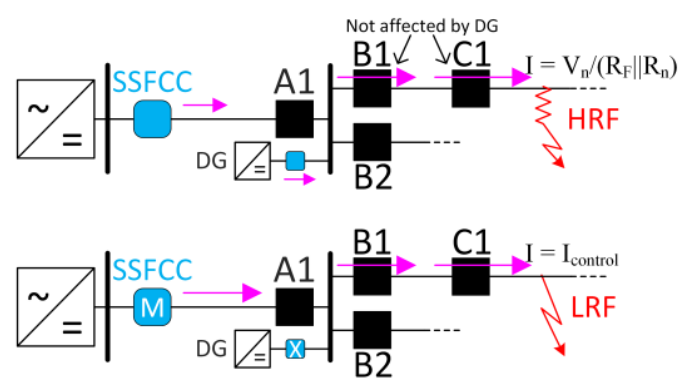

Fig. 17. Participation of DGs.

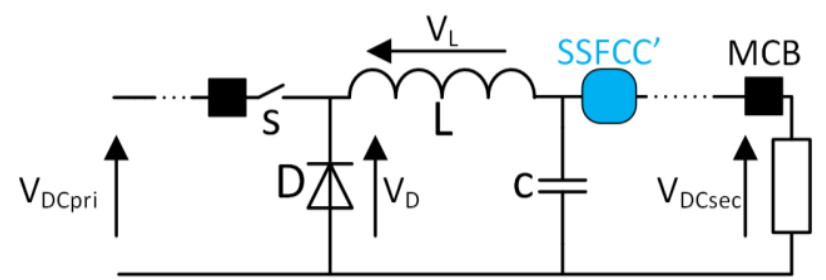

Fig. 18. Typical configuration of a buck converter.

\section{B. Participation of DGs}

To accommodate distributed generators (DG) on LVDC networks, a supplementary protection function can be added to ensure the MLE protection operates as intended. The participation of DGs must maximally maintain the inherent fault current through the downstream MCB-relays within the fault path. As shown in Fig. 17, during HRF conditions, the fault current through the near-fault MCB-relays (B1 and C1) is determined from the nominal voltage and its downstream equivalent resistance only. Since the voltage drop across the primary feeder is still negligible during HRF conditions, the DG will have no impact on the current through the downstream MCB-relays. However, to accommodate LRF conditions and avoid the potential for the DG contributing additional fault current, the DG could employ a fast-speed breaker to block its current infeed quickly to minimize the impact to the MCBrelays' operating time. This may be implemented using a gridside under-voltage detection threshold mechanism.

\section{Connection with downstream DC-DC Converters}

DC-DC converters can provide a supply to independent radial DC networks. Such converters, such as the topology shown in Fig. 18, employ filter capacitors that may rapidly discharge during short-circuit fault conditions. To mitigate this issue, an SSFCC may also be used to impede high fault current to avoid fully discharging the capacitor. Accordingly, protection coordination can be realized using the same methodology presented in the previous sections. If the DC-DC converter also features a capacitive filter on the input side to the converter, a fast-acting SSCB can be employed to prevent excessive current contributions from this to the wider grid under LRF conditions (similar to the proposed approach for DGs).

\section{FUTURE WORK}

Future iterations of the SSFCC design will include appropriate snubber elements and a closed-loop fault current controller. To minimize the induced voltage across the SSCB, soft turn-off with ramp current decay may be deployed to avoid transient over-voltage. Since the fault current response of a real DC power distribution system is unpredictable, a dynamic closed-loop current controller may be implemented to realise more accurate average fault current let-through.

Additionally, hardware designs of the SSCB for current blocking during LRF conditions and the reclosers for loop-type protection will be considered and verified experimentally.

\section{CONCLUSION}

This paper has reviewed recently proposed high-speed DC protection schemes and analyzed their limitations. These types of schemes are theoretically effective but require a large quantity of high-speed measurement devices, processors and SSCBs to function. Accordingly, they are expensive to implement practically. Furthermore, the sub-millisecond decision-making time-window has a negative impact on protection stability and effective coordination. Such schemes may only be suitable for advanced compact DC networks, such as aircraft and shipboard electrical systems.

For grid application DC distribution networks, a DC-version overcurrent protection scheme based on the "FCL+MCBs" structure has been proposed that provides effective coordination within a moderate operating speed. This is achieved by coordinating one fast current-limiting upstream device and a number of downstream moderate operating speed voltagedependent breakers. This solution requires no communication and significantly less high-speed devices, which will not only dramatically reduce the expense but also improve its reliability by widening the decision-making time-window. Additionally, the MLE protection scheme provides flexibility in network extension. The original protection layout needs no reprogramming when a new feeder is installed. Though the operating time is longer, the energy dissipation will not dramatically rise because of the use of current limitation.

More generally, this paper has shown that there is a timely opportunity to study the metrological limitations more thoroughly as DC microgrid research matures, and ensure innovations in this field are accessible to new microgrid scheme planners. In particular, the authors believe that further specific research into moderate-speed DC protection schemes could 
enable a more rapid uptake of widespread large-scale civil DC microgrid applications.

\section{REFERENCE}

[1] T. Dragicevic, X. Lu, J. Vasquez and J. Guerrero, "DC microgrids - part II: a review of power architectures, applications, and standardization issues," IEEE Trans. Power Electron., vol. 31, no. 5, pp. 3528-3549, May 2016.

[2] N. Mohan, T. Undeland and W. Robbins, "Line-frequency phasecontrolled rectifiers and inverters: line-frequency ac-controlled dc," in Power Electronics: Converters, Applications, and Design, $3^{\text {rd }}$ ed. USA: John Wiley \& Sons, inc., 2003, ch. 5, pp. 103-111.

[3] H. Ergun, and D. V. Hertem, "Comparison of HVDC and HVDC technologies," in HVDC Grid: For Offshore and Supergrid of the Future, Wiley-IEEE Press, 2016, ch. 4, pp. 79-96.

[4] J. Yang, J. Fletcher, J. OReilly, "Short-circuit and ground fault analyses and location in VSC-based DC network cables," IEEE Trans. Ind. Electron., vol. 59, no. 10, pp. 3827-3837, Oct. 2012.

[5] D. Salomonsson, L. Soder, and A. Sannino, "Protection of lowvoltage DC microgrids," IEEE Trans. Power Del., vol. 24, no. 3, pp. 1045-1053, Jul. 2009.

[6] S. Fletcher, P. Norman, K. Fong, S. Galloway, and G. Burt, "Highspeed differential protection for smart DC distribution system," IEEE Trans. Smart Grid, vol. 5, no. 5, pp. 2610-2617, 2014.

[7] S. Fletcher, "Protection of physically compact multi-terminal DC power system," Ph.D. thesis, Dept. Elect. Eng., Strathclyde Univ., Glasgow, UK, pp. 54-55, 2013.

[8] K. Satpathi, A. Ukil, J. Pou, "Short-circuit fault management in DC electric ship propulsion system: protection requirements, review of existing technologies and future research trends," IEEE Trans. Transport. Electrific., vol. 4, no. 1, pp. 272-291, 2018.

[9] D. Tzelepis, A. Dysko, G. Fusiek, J. Nelson, P. Niewczas, D. Vozikis, P. Orr, N. Gordon and C. Booth, "Single-ended differential protection in MTDC network using optical sensors," IEEE Trans. Power Del., vol. 32, no. 3, pp. 1605-1615, June 2017.

[10] M. Baran and N. Mahajan, "Overcurrent protection on voltagesource-converter-based multi-terminal dc distribution systems," IEEE Trans. Power Del., vol. 22, no.1, pp. 406-412, Jan. 2007.

[11] A. Emhemed, and G. Burt, "An advanced protection scheme for enabling an LVDC last mile distribution network," IEEE Trans. Smart Grid, vol. 5, no. 5, pp. 2602-2609, Sep. 2014.

[12] M. Monadi, C. Gavriluta, A. Luna, J. Candela and P. Rodriguez, "Centralized protection strategy for medium voltage DC microgrids," IEEE Trans. Power Del., vol. 32, no. 1, pp. 430-440, Feb. 2017.

[13] X. Feng, L. Qi, and J. Pan, "A novel fault location method and algorithm for DC distribution protection," IEEE Tran. Ind. Appl., vol. 53, no. 3, pp. 1834-1840, May 2017.

[14] R. Li, L. Xu, and L. Yao, "DC fault detection and location in meshed multi-terminal HVDC systems based on DC reactor voltage change rate," IEEE Trans. Power Del., vol. 32, no. 3, pp. 1516-1526, June 2017.

[15] A. Meghwani, S. Srivastava and S. Chakrabarti, "A Non-unit Protection Scheme for DC Microgrid Based on Local Measurements," IEEE Trans. Power Del., vol. 32, no. 1, pp. 172181, Feb. 2017.

[16] C. Li, P. Rakhra, P. Norman, P. Niewczas, G. Burt, and P. Clarkson, in IEEE $2^{\text {nd }}$ International Conference on DC Microgrid, pp. 153-159, 2017.

[17] C. Li, P. Rakhra, P. Norman, G. Burt and P. Clarkson, "Metrology requirements of state-of-the-art protection schemes for DC microgrids," in IET $14^{\text {th }}$ International Conference on Developments in Power System Protection, 2018.

[18] P. Cairoli, R. Rodrigues and H. Zheng, "Fault current limiting power converters for protection of DC microgrid," in SoutheastCon, 2017.
[19] X. Y. Chen and J. X. Jin, "Energy efficiency analysis and energy management of a superconducting LVDC network," IEEE Trans. Appl. Supercond., vol. 26, no. 7, Oct. 2016, Art. no. 5403205.

[20] L. Qi, J. Pan, X. Huang, and X. Feng, "Solid-state fault current limiting for DC distribution protection," in IEEE Electric Ship Technologies Symposium, pp. 187-191, 2017.

[21] M. Bamber, et al., "Overcurrent protection for phase and earth fault," in Network protection and automation guide: protective relays, measurement and control, $1^{\text {st }}$ ed. vol. 9, Alstom Grid, 2011, pp. 1-31.

[22] P. Rakhra, "On the protection of compact DC power systems with high-power energy storage," Ph.D. thesis, Dept. Elect. Eng., Strathclyde Univ., Glasgow, UK, pp. 90-94, 2017.

[23] Semikron, Power MOSFET Modules - Semitrans TM M1 SKM 111AR Datasheet, [Online]. Available: http://www.gdrectifiers.co.uk/uploads/SEMIKRON_DataSheet_ SKM_111_AR_.pdf

[24] LEM, Current Transducer-HAS 50..600-S and Voltage Transducer-LV 25-P, [Online]. Available: www.lem.com

[25] National Instruments, Intelligent Real-Time Embedded Controller for CompactRIO on FPGA Chassis and I/O modules, [Online]. Available: www.ni.com/pdf/manuals 